\title{
THE DESIGN AND SIMULATION OF ROSE HARVESTING ROBOT
}

\section{YERRAPOTHU MANIKANTA ${ }^{1}$, SEERAM SRINIVASA RAO ${ }^{2} \&$ R. VENKATESH ${ }^{3}$}

${ }^{1}$ Student, Department of Mechanical Engineering, Koneru Lakshmaiyya Education Foundation,

Vaddeswaram, Vijayawada, Guntur, Andhra Pradesh, India

${ }^{2}$ Professor, Department of Mechanical Engineering, Koneru Lakshmaiyya Education Foundation,

Vaddeswaram, Vijayawada, Guntur, Andhra Pradesh, India

${ }^{3}$ Assistant Professor, Department of Mechanical Engineering, Koneru Lakshmaiyya Education Foundation,

Vaddeswaram, Vijayawada, Guntur, Andhra Pradesh, India

\section{ABSTRACT}

The agriculture sector is backbone for several country economies; the economic status depends on the agriculture of those countries. If we increase the ease in agriculture process, with latest technologies through automation in processes such as cultivating, harvesting, spraying pesticides etc., it increases the growth of the economic status of the country by raising its GDP. Rose flower is one, having high demand in the society but the problem with this flower is its harvesting. Rose flower cultivation is one of the difficult methods, as to harvest the thorns on their stems cause's injuries and a training is required for the labours to learn harvesting. Decreasing the labour population day by day due to various problems increases the demand of labour and their cost. Due to the training and high labour costs in rose flower cultivation tends to increase in its production cost. To solve all these problems a rose harvesting machine which is fully automated is designed and it works with machine learning algorithms and image processing tool designed in Matlab.

KEYWORDS: Rose Harvesting Robot, Nä̈ve Bayes Algorithm, Image Acquisition, Agricultural Robot \& Flower Cutting Robot

Received: Nov 28, 2018; Accepted: Dec 18, 2018; Published: Jan 04, 2019; Paper Id.: IJMPERDFEB201919

\section{INTRODUCTION}

Rose flower is a popular and profitable flower having high demand in the world. An intensive care is required to obtain quality of flowers [1]. So it employs many people to be engaged in rose cultivation. But the problem with the rose flower harvesting and labour wages, because of some reasons like people are interested in developing their lives by getting education and another reason is that they are facing a lot of difficulties like rose thorns keep hurting them physically, clothes were also affected and they have to keep their posture in bending position facing the ground for long hours for the harvesting of flowers which leads them to severe pain in their backbone, leg, thigh and feet etc., by making them fatigue[2]. This tends to decrease in man power and increase in labour costs.

Rose flower is a valuable product in the market. So, they are grown in greenhouse to collect highest quality flowers all over the year. Harvesting is an important process and it has to be performed by a skilled employee with high accuracy above the first, second or third eyes of rose stem depending on the thickness of the stem [3]. The efficiency and prediction levels of employees differs from one person to another and for one 
individual it also differs from morning first hour to evening last hours. Some may harvest properly bloomed roses and some may not. Natural interruptions like heavy rain, fog and mist etc. may also occur while harvesting is in process. Automation can give better results than human employees in such conditions [4]. Due to human limitations, limited working hours, interruptions during the work hours it is better to employ robots to do the task since they can do the work repeatedly without getting tired for longer hours. Also the prediction whether a rose has blossomed or not can be programmed correctly through computer programs and the robot can detect the correct colour, size and texture of the rose and identified the fully grown roses for harvesting[5]. Harvesting is composed of four stages which are detecting and locating harvestable rose bud, determining cut location, cutting the stem, and transporting stem and rose flower to the storage box [6].

The harvesting should be carried out without human intervention, when we start the system then it has to go autonomously in to the fields with the help of camera's live-streaming video and has to pluck the flowers by the manipulator with its end effect or, the robot will take input i.e. path finding code with obstacle avoidance through a programme by controller and goes into the path up to rose plant. In some regions rose cultivation is done in pots. In this case a stationary robot is used and the rose pots will come towards the robot by moving conveyors [7]. When the rose flower was recognised by camera then robot will stop at that plant and image processing algorithms [9] will run the code as per the given specified dataset by training and testing process to detect the roses by image segmentation process and machine learning algorithms like logistic regression and Naive Bayes Algorithms[12], if the flower was fully blossomed then it gives the signal to the manipulator to collect the flower with specially designed end-effector or if not it moves to the other. The end-effector has to cut the flower above the second or third eyes from the sepal toward the roots, and put them in to the storage box. Such that it can harvest for longer hours of the day and night, even in uneven weather conditions to increase productivity with low expenditure. This type of robot is designed in CREO and coding for path planning was done in KEIL software and the path planning code is simulated in the PROTEUS professional suite, the machine learning algorithms were done with python in spider in anaconda navigator, the image processing is done in MATLAB software and static analysis is done in ANSYS software.

\section{METHODOLOGY}

\subsection{Object Recognition by Image Processing}

Image processing plays a predominant role in this system from the starting to till the end of harvesting, the process will start by training the neural network from CIFAR-10 Dataset to identify the objects in the images captured by camera. This image processing with the computer algorithms in MATLAB are called Digital image processing. It helps in converting the signal from the camera in to digital images, improve quality by removing noise, extracting size, scale or number of objects in scenes and preparing images for better identification. The rose stems are traced by separating the image into a large number of small pixels and analysing the separated pixels to know the object in the scene. The process of separating is called image segmentation and is done by using thresh holding and morphological operations.

The process starts by reading the video input from the camera and taking the snapshot by using the get snapshot command. Convert the image to black and white in order to prepare for boundary tracing by thresh holding. After thresh holding, a binary image is obtained. Removing the noise pixels which aren't belonged to object of interest in binary image by using morphological functions. Finding the boundaries of objects in the scene by using boundaries and label commands. The following Figure 1 ( $a, b, c, d$ ) Contains original image, binary image, noise removed images and boundary tracing 
image.

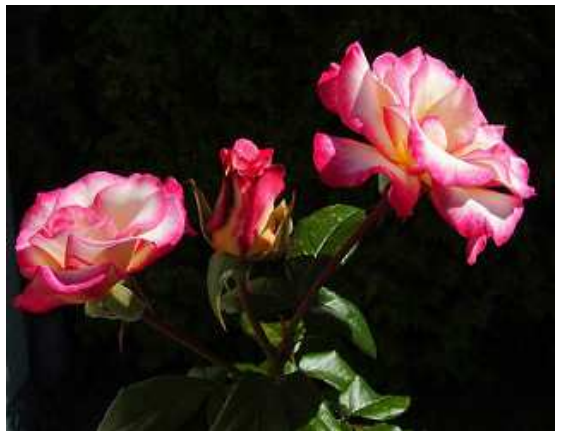

Figure 1 (a): Captured Input from Camera

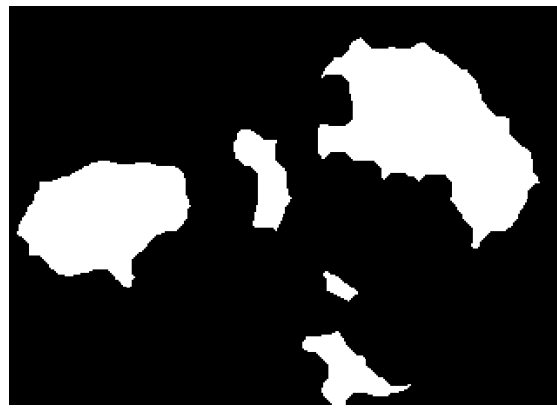

Figure 1 (c): Noise Removed Image

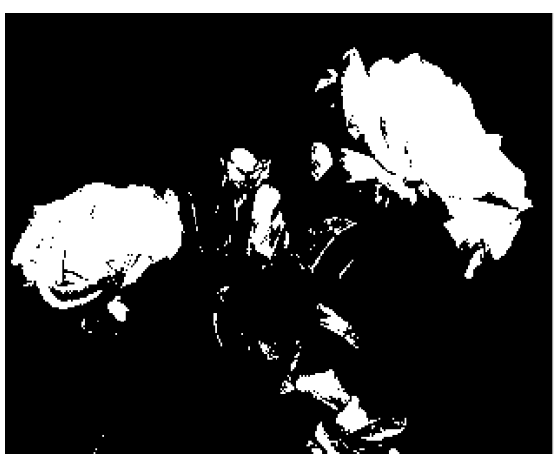

Figure 1 (b): Grey Scale Binary image

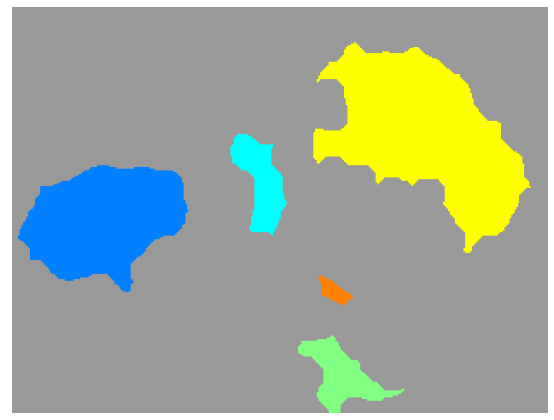

Figure 1 (d): Boundary Tracing Image

\subsection{Dataset Preparation}

A rose dataset is prepared according to the specifications like sepal length, sepal width, petal length, petal width with the reference of fisher's iris dataset [8]. The dataset will look like 150 by 5 matrices having 150 samples of each specification and the fifth column consists of five different shapes, so that each shape consists of 30 samples of data. From the dataset, first two samples of each shape is shown in table 1

Table 1: Sample Dataset

\begin{tabular}{|c|c|c|c|c|l|}
\hline Id & $\begin{array}{c}\text { Sepal Length } \\
\text { in Cm }\end{array}$ & $\begin{array}{c}\text { Sepal Width } \\
\text { in Cm }\end{array}$ & $\begin{array}{c}\text { Petal Length } \\
\text { in Cm }\end{array}$ & $\begin{array}{c}\text { Petal Width } \\
\text { in } \mathbf{C m}\end{array}$ & \multicolumn{1}{|c|}{ Shapes } \\
\hline 1 & 5.1 & 3.5 & 1.4 & 0.2 & cupped bloom \\
\hline 2 & 4.9 & 3 & 1.4 & 0.2 & cupped bloom \\
\hline 31 & 4.8 & 3.1 & 1.6 & 0.2 & globular bloom \\
\hline 32 & 5.4 & 3.4 & 1.5 & 0.4 & globular bloom \\
\hline 61 & 5 & 2 & 3.5 & 1 & high-centred \\
\hline 62 & 5.9 & 3 & 4.2 & 1.5 & high-centred \\
\hline 91 & 5.5 & 2.6 & 4.4 & 1.2 & rosette bloom \\
\hline 92 & 6.1 & 3 & 4.6 & 1.4 & rosette bloom \\
\hline 121 & 6.9 & 3.2 & 5.7 & 2.3 & pompon bloom \\
\hline 122 & 5.6 & 2.8 & 4.9 & 2 & pompon bloom \\
\hline
\end{tabular}

\subsection{Naïve Bayes Algorithm}

Naïve Bayes Algorithm is a set of classification algorithms based on Bayes' Theorem. Bayes' Theorem finds the probability of an event occurring given the probability of another event that has already occurred [11]. Bayes' theorem is stated mathematically as the following equation: 
$P(C / D)=\frac{P(D / C) / P(C)}{P(D)}$

In simple terms, a Naive Bayes classifier assumes that the presence of a particular feature in a class is unrelated to the presence of any other feature. There are different types of Naïve Bayes Algorithms are there, they are

- Gaussian Naive Bayes,

- Multinomial Naive Bayes.

- Bernoulli Naive Bayes.

In the above types, the Gaussian Naïve Bayes Algorithm is used to predict whether the flower is fully blossomed are not with the help of prepared dataset.

\section{SOLID MODEL DESIGN}

The solid model of the robot was designed in the solid modelling software CREO which is also known as PRO-E. The model looks like a mini-car like vehicle having four wheels with four DC-motors, so that it can turn easily at the row ends. It has four hydraulic cylinders to give to and fro motion vertically to pluck the flowers which are at the beyond limits of the robot i.e. some of the rose plants may grow taller than normal height. In such conditions these hydraulic cylinders plays an important role by lifting the robot upward to collect the taller plant roses. The 3D-assembly drawing of the rose harvesting robot is shown in Figure 2.

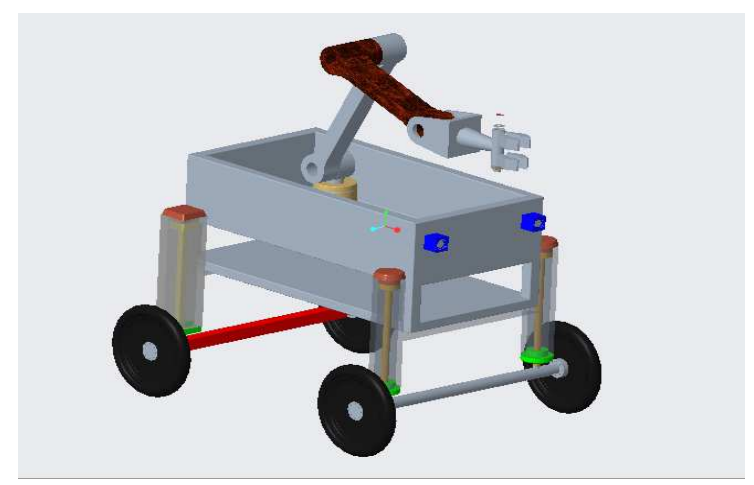

Figure 2: 3D-Design of Rose Harvesting Robot

One of the main parts of the robot is its body which also acts as container i.e., the collected roses are stored in this container, also called as collecting basket, an empty space is given under this container body for placing controllers, sensors and required wirings.

A manipulator with RRR(Articulated Arm) is located in the container at one corner with having three links, in which first one is connected to the container body with rotary joint, second one is connected to first link with revolute joint and the third link is connected to second one with revolute joint, the manipulator is designed to fit in the container when it is in closed position i.e. the angle between link 1 and link 2 is 90 degrees and the angle between link 2 and link 3 is zero degrees and parallel to each other. The first link is provided with hydraulic cylinder to move up and down to fit the whole arm in to the container. We can call this position as rest position and it is shown in Figure 3. 


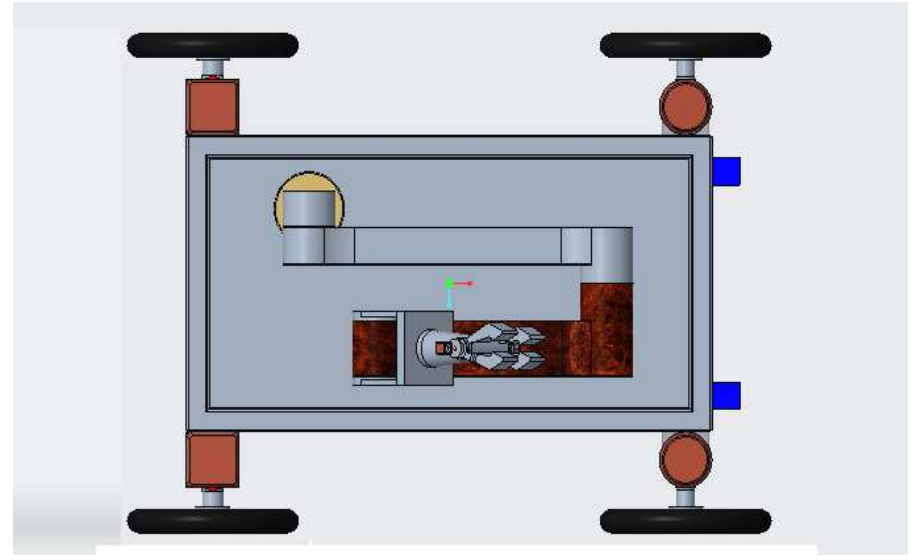

Figure 3: Top View of the Robot when the Manipulator is in Rest Position

An end-effector is designed specially for the flower harvesting and looks like tongs and it is shown in Figure 4.

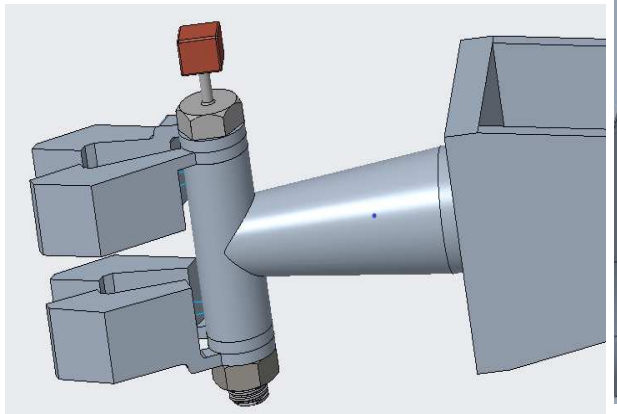

Figure 4 (a): End-Effector Side View

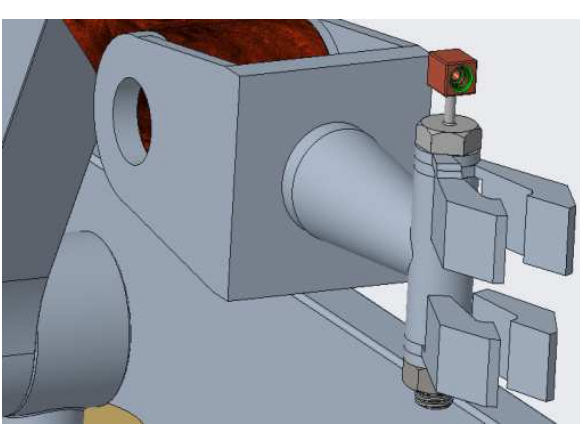

Figure 4 (b): Orthogonal View

The figure shows two sets of tongs, in which the upper set is made for holding the rose stem smoothly as per the signal given by the controller and the lower set is made for cutting. An adjustable camera is placed above the bolt to show the rose stem, we can adjust the camera to middle space in between the two set of tongs. Two motors are required to control the end-effector.

\section{ANALYSIS OF DESIGNED SOLID MODEL}

Structural static analysis was done on the designed model to know the ability of the robot for different loads acting at different positions on the system, the total deformation of the robot is from the free end to fixed position is shown in Figure 5.

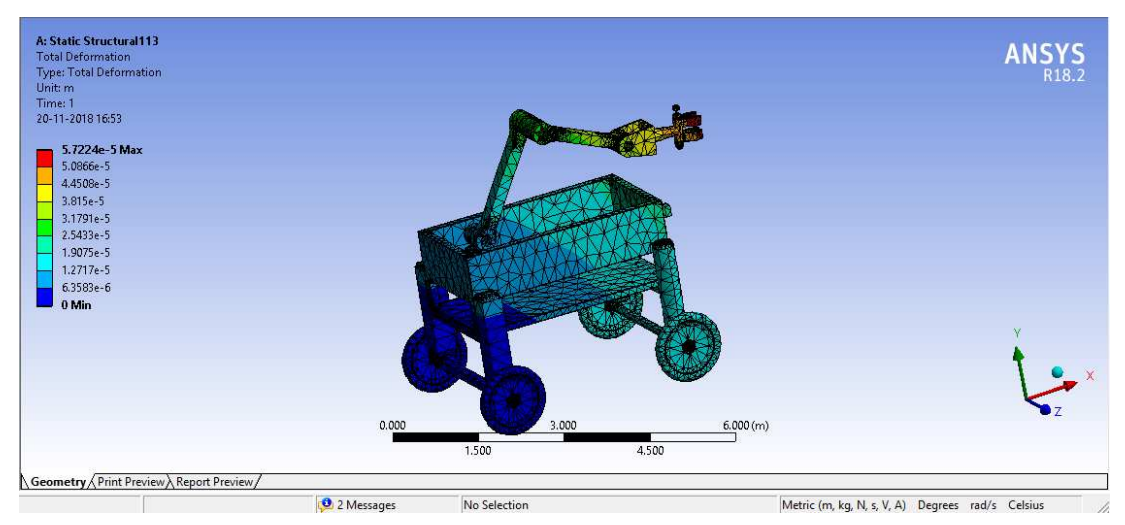

Figure 5: Total Deformation of the Solid Model 


\section{PATH CONTROL}

The path was planned according to the layout of the field, generally the path will be like straight lines with U-turns at the end of that row and enters into the second row. The path planning algorithm was programmed in KEIL software which is programming software looks like c-programming. The required components are DC-motors, L293D IC driver, AT89C52 from 8052 family controller and required switches and connection wires.

The connections with the above mentioned components are done in PROTEUS design suite software and the connected circuit was simulated by debugging the program from KEIL to AT89C52 in. HEX file format, the simulated circuit was shown in the Figure 6.

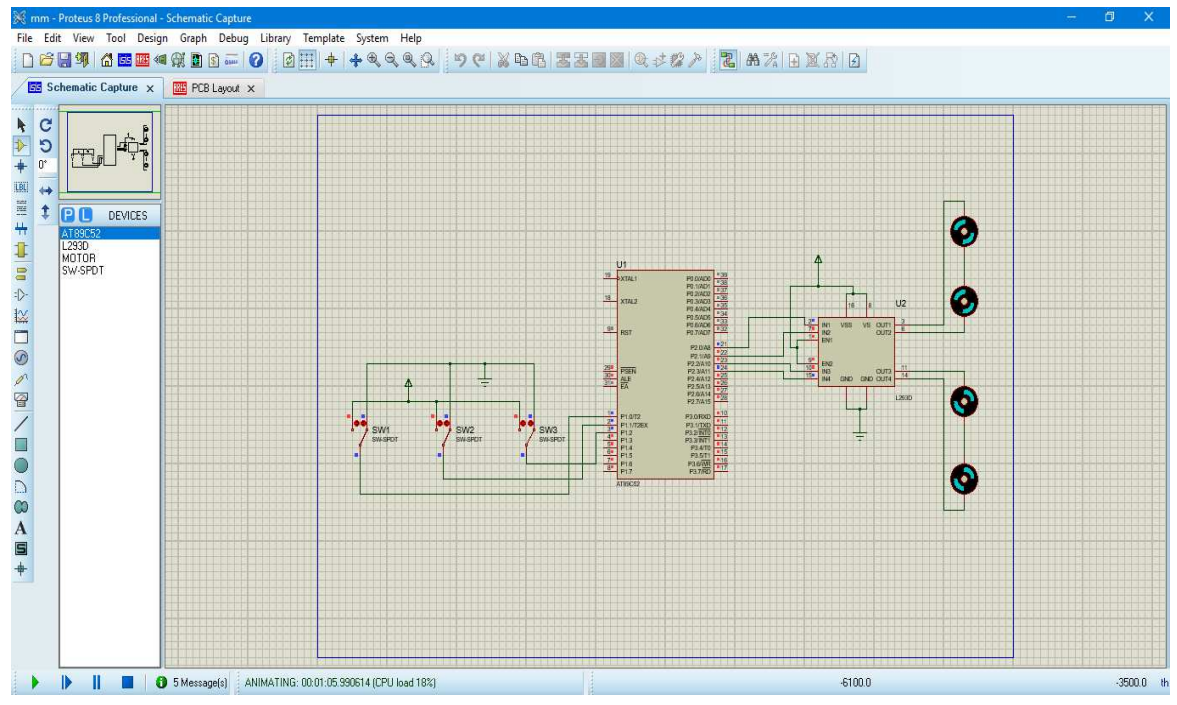

Figure 6: Path Control Simulation in PROTEUS Software

The code was written in KEIL software for running the system from one point to another with avoiding the obstacles as per video streaming input given by the camera. Path control means controlling the path i.e., when system was started then the camera will capture the path and give continuous video stream to the controller, this controller analyses the captured video as per the written code and gives the signal to the motors to start and run if there was no obstacle in front of the vehicle. If suddenly an obstacle was captured then the controller gives a signal to motors to stop and take left or right turn, the turning was done by running one side motors in reverse.

\section{RESULTS AND DISCUSSIONS}

Using the prepared dataset classification and regression algorithms are performed for classifying the shapes and to know whether the flower is fully blossomed are not. The process is done with the PYTHON script in SPYDER IDE by ANACONDA Navigator.

The process starts with saving the running python file and dataset file in same folder and importing pandas, matplotlib, sea born for plotting graphs and importing K Neighbours Classifier, Logistic Regression and Test train split algorithms from Scikit learn package followed by importing dataset in csv format because pandas is designed to read the.csv file only. After reading the dataset the layout is shown in output. By using pair plot from the sea born library, the sixteen plots with each specification on $\mathrm{x}$-axis and $\mathrm{y}$-axis to know how rose flowers are varying according to the shapes in five different colours with these samples is shown in Figure 7. 
RangeIndex: 150 entries, $\theta$ to 149 Data columns (total 6 columns):

Id 150 non-null int64

SepallengthCm 150 non-null float64

SepalWidthCm 150 non-null float64 PetallengthCm 150 non-null float64 PetalWidthCm 150 non-null float 64 shapes 150 non-null object

dtypes: float64(4), int64(1), object(1) memory usage: $7.1+\mathrm{KB}$

shapes

- cupped bloom

- globular bloom

- high-centred

- rosette bloom

- pompon bloom
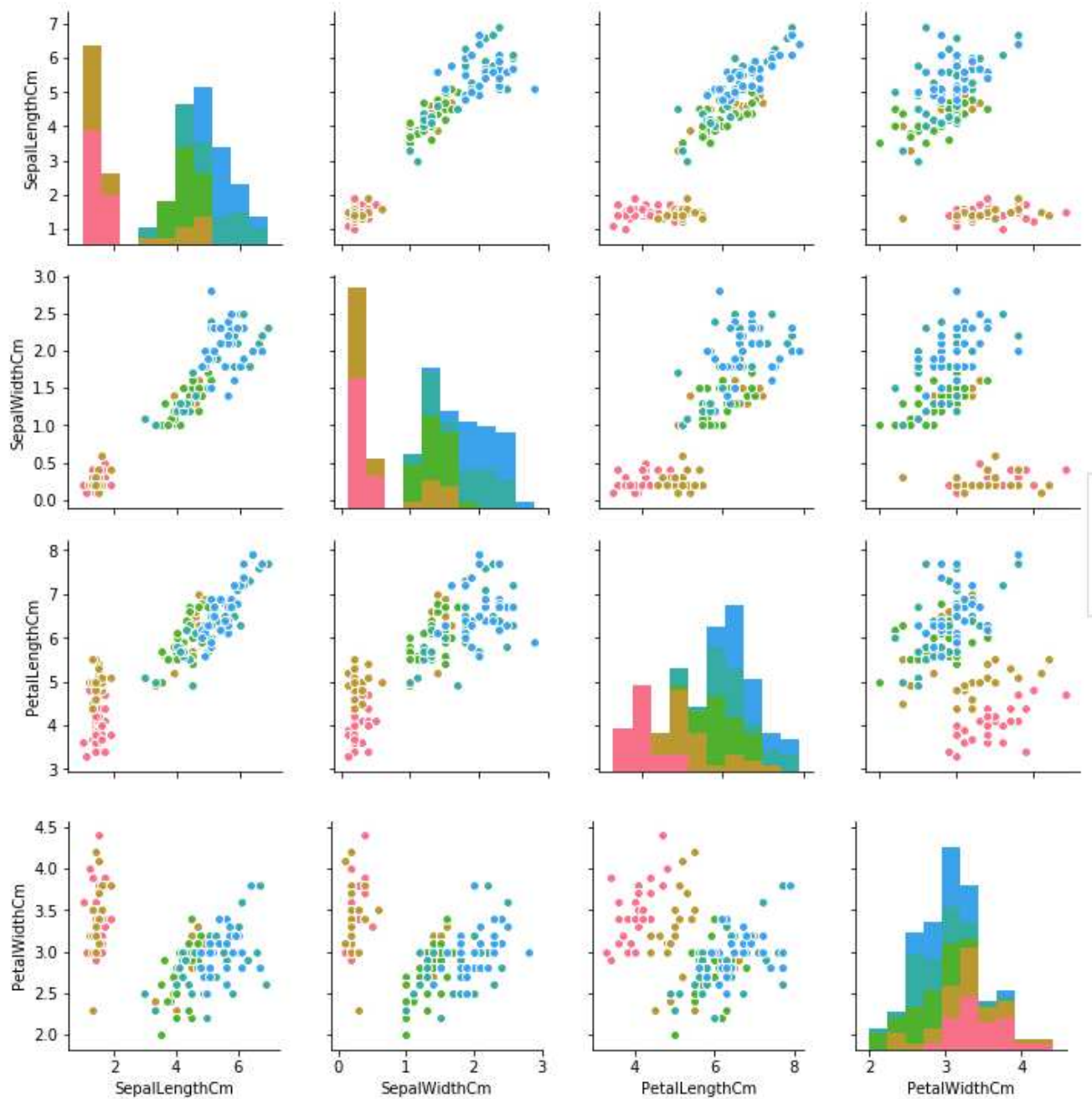

Figure 7: Pair Plot of Dataset according to Shapes with Four Specifications Each Other

In the above plot we have seen all the specifications in one graph, but for better understanding four single graphs with a single specification on $\mathrm{x}$-axis and shapes on $\mathrm{y}$-axis is drawn using violin plot from seaborn library package. Each shape loop variance region of flowers according to the sepal length on $\mathrm{x}$-axis and shapes on $\mathrm{y}$-axis is shown in Figure 8. 


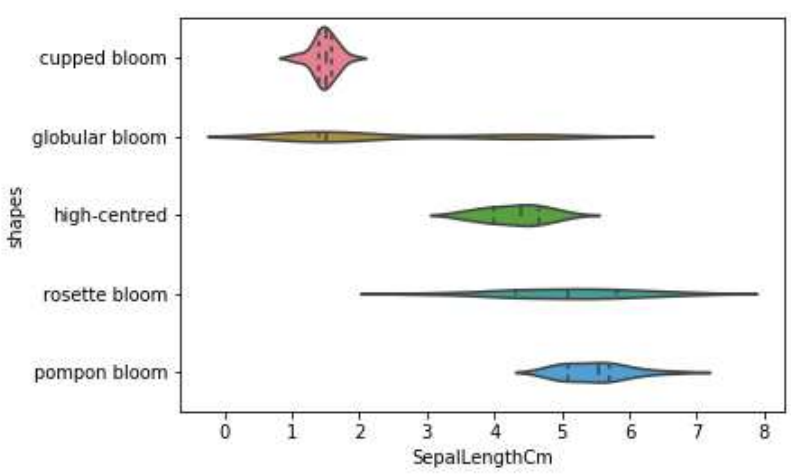

Figure 8: Plot between Sepal Length and Shapes

The loop variance region of the shapes of the flowers according to the sepal width is shown in Figure 9.

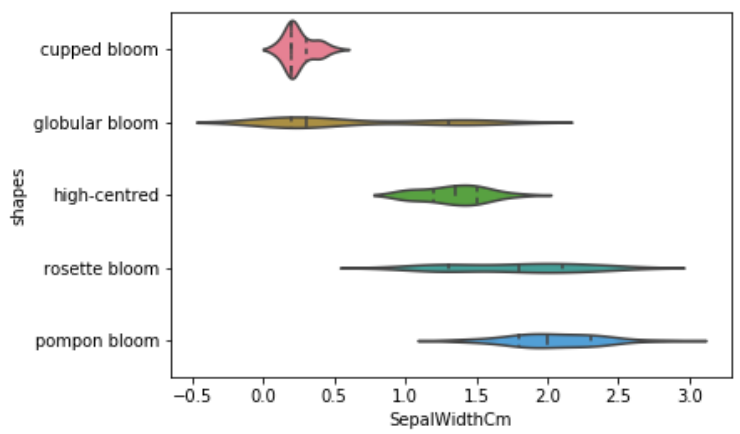

Figure 9: Plot between Sepal Width and Shapes

The loop variance region of the shapes of the flowers according to the petal length is shown in Figure.10.

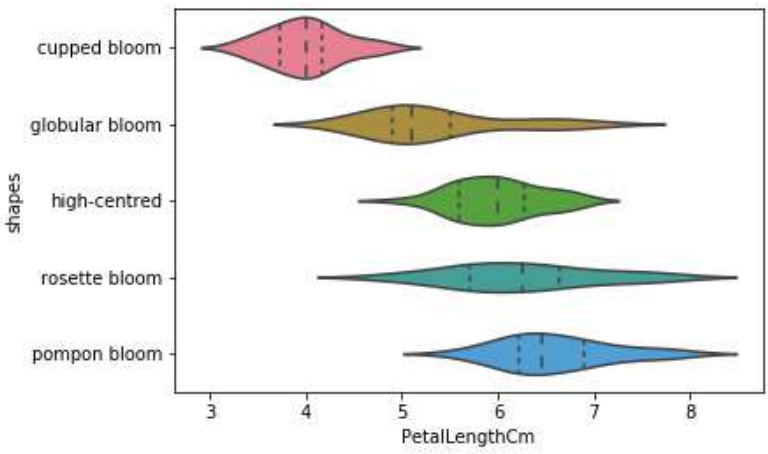

Figure 10: Plot between Petal Length and Shapes

The loop variance of the shapes of the flowers according to the petal width is shown in Figure 11. 


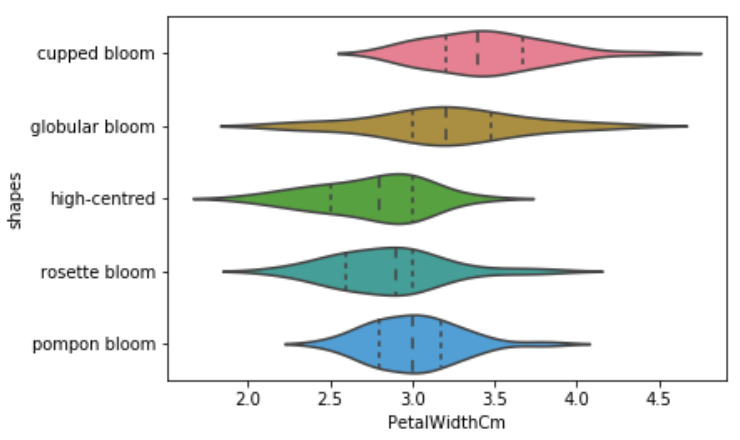

Figure 11: Plot between Petal Width and Shapes

The Gaussian Naïve Bayes classification algorithm starts analysing the input scenes having rose flower with the specifications in dataset and plotted graphs and predicts the flower in to two conditions i.e., blossomed flower is assigned to ' 1 ', non-blossomed flower is assigned to ' 0 '. If the output from the algorithm is ' 1 ' then the manipulator has to cut the flower, if the output is ' 0 ' then manipulator leave's that flower and moves to the another flower. The accuracy of this algorithm is 75 to $80 \%$ approximately and best suit for rose harvesting.

\section{CONCLUSIONS}

The proposed rose harvesting robot is designed with image acquisition by using colour segmentation and thresh hold for object recognition. The Gaussian Naïve Bayes algorithm uses the prepared dataset to predict blossomed rose flowers. Using the advanced learning algorithms and programming them with powerful tools enables the Automation process in Rose Harvesting. The efficiency of these algorithms were the key factors for complete automation in Agriculture. A lot of research is happening in the field of Data Science which enables the effective procedure of Automation in the fields of harvesting and nurturing various crops which in turn yields better revenue for the farmers and raise the GDP of the nation.

\section{ACKNOWLEDGEMENTS}

The authors are grateful to the support of the K L E F (Deemed to be University), Dept. of Mechanical Engineering, FIST sponsored Advance Prototyping and Manufacturing Lab (SR/FST/ETI-317/2012(C)) for supporting us throughout the project.

\section{REFERENCES}

1. Reid, Aileen. "Greenhouse roses for cutflower production." (2008).

2. Abarna, J., and A. Arockia Selvakumar. "Rose flower harvesting robot." International Journal of Applied Engineering Research 10, no. 55 (2015): 4216-4220.

3. Gürel, Cahit, and Abdulkadir Erden. "Conceptual Design of a Rose Harvesting Robot for Greenhouses." In Proc. of The 20th Int. Conf. on Mechatronics and Machine Vision in Practice-M2ViP, pp. 18-20. 2013.

4. Bachche, Shivaji. "Deliberation on design strategies of automatic harvesting systems: A survey." Robotics 4, no. 2 (2015): 194-222.

5. Charu Bhartiya, Prof. Ashish P” Image Processing Based Rose Harvesting System using Raspberry Pi”, Vol. 4 Issue 04, April-2015, ISSN: 2278-0181 
6. GÜREL, Cahit, M. Hassan GM ZADEH, and Abdulkadir ERDEN. "Development and Implementation of Rose Stem Tracing using a Stereo Vision Camera System for Rose Harvesting Robot."

7. Kumar, Gurram Narendra Santosh, and A. Srinath. "Exploration of Accelerating Moving Walkway for Futuristic Transport System in Congested and Traffical Areas." (2018): 616-624.

8. Fisher, Ronald A., and Michael Marshall. "Iris data set." RA Fisher, UC Irvine Machine Learning Repository 440 (1936).

9. Parker, Jim R. Algorithms for image processing and computer vision. John Wiley \& Sons, 2010.

10. Kohan, Armin, Ali Mohammad Borghaee, Mehran Yazdi, Saeid Minaei, and Mohammad Javad Sheykhdavudi. "Robotic harvesting of rosa damascena using stereoscopic machine vision." World Applied Sciences Journal 12, no. 2 (2011): 231-237.

11. Leung, K. Ming. "Naive bayesian classifier." Polytechnic University Department of Computer Science/Finance and Risk Engineering (2007).

12. Danthala, S., et al. "Robotic Manipulator Control by using Machine Learning Algorithms: A Review." International Journal of Mechanical and Production Engineering Research and Development, vol. 8, no. 5, 2018, pp. 305-310. SCOPUS, www.scopus.com, doi:10.24247/ijmperdoct201834. 